\title{
ENTROPY AND DENSITY
}

\author{
GEORGE H. STEIN
}

ABstract. We prove that for any $r$, "entropy $=r$ " is a dense condition in the uniform topology.

In 1959, Rohlin [5] proved the set of transformations with zero entropy is a dense $G_{\delta}$ in the uniform topology. See Parry [3, p. 103] for a proof. In this note, we prove:

THEOREM. For each $r, \infty \geqq r>0$, the set of transformations with entropy equal to $r$ is dense in the uniform topology.

For the weak topology, the analogous theorem can be proved easily with the aid of the conjugacy lemma (Halmos [2, pp. 77-78]): the conjugacy class of an antiperiodic transformation is dense. To complete the proof requires only the existence of antiperiodic transformations with arbitrary entropy (Lemma 1, below). The conjugacy class of an antiperiodic transformation is never dense in the uniform topology. However, a simple lemma enabling one to compute the entropy of a transformation in terms of its entropy on invariant pieces provides the key.

More precisely, we consider the unit square with Lebesgue measure; transformations which are bijections, measurable together with their inverses, and measure-preserving. (The results are true for Lebesgue spaces with nonatomic measure, but we choose the unit square for ease in picturing a certain construction.) The uniform topology on transformations is given by the metric: $d(S, T)=m(\{x: S x \neq T x\})$. See [2, pp. 61-68] for more information.

Computation Lemma. Let $T: I^{2} \rightarrow I^{2}$ be a transformation with $\{M, N\}$ a measurable partition, each element of which is invariant. Then

$$
h(T)=h\left(T_{m}\right) \mid+h\left(\left.T\right|_{N}\right) .
$$

Notation. denotes restriction, capital Greek is used for partitions, $\checkmark$ denotes smallest common refinement, $h$ is the entropy function, etc. as in Billingsley [1].

REMARK. This follows from the more general formula of Rohlin $[4$, p. 28]. However, a direct proof is easy.

Received by the editors June 4, 1970 .

AMS 1970 subject classifications. Primary 28A70.

Key words and phrases. Entropy. 
Proof. $h(T)=\sup _{\Gamma} h(\Gamma, T)$ by definition. If $\Gamma \subset \Lambda, h(\Gamma, T) \leqq h(\Lambda, T)$. So, noting that $\Gamma \subset \Gamma \vee\{M, N\}$, without loss of generality, we may assume that all partitions refine $\{M, N\}$. Now

$$
\begin{aligned}
h(\Gamma, T)= & \lim _{n \rightarrow \infty} \frac{1}{n} H\left(\Gamma \vee \cdots \vee T^{n-1} \Gamma\right) \\
= & \lim _{n \rightarrow \infty} \frac{-1}{n} \sum_{A \in \Gamma \vee \cdots \vee T^{n-1} \Gamma} m(A) \log (m(A)) \\
= & \lim _{n} \frac{-1}{n} \sum_{A \in\left(\Gamma \vee \ldots \vee T^{n-1} \Gamma\right) \cap M} m(A) \log (m(A)) \\
& +\lim _{n} \frac{-1}{n} \sum_{A \in\left(\Gamma \vee \ldots \vee T^{n-1} \Gamma\right) \cap N} m(A) \log (m(A)) \\
= & \lim _{n} \frac{1}{n} H\left(\left.\Gamma \cap M \vee \cdots \vee T\right|_{M} ^{n-1}(\Gamma \cap M)\right) \\
& +\lim _{n} \frac{1}{n} H\left(\left.\Gamma \cap N \vee \cdots \vee T\right|_{N} ^{n-1}(\Gamma \cap N)\right) \\
& \left(\operatorname{since}\left(\Gamma \vee \cdots \vee T^{n-1} \Gamma\right) \cap M=(\Gamma \cap M) \vee\right. \\
= & h\left(\Gamma \cap M,\left.T\right|_{M}\right)+h\left(\Gamma \cap N,\left.T\right|_{N}\right) .
\end{aligned}
$$

As $\Gamma$ ranges over refinements of $\{M, N\}, \Gamma \cap N$ and $\Gamma \cap M$ range over partitions of $N$ and $M$ respectively. Hence, by positivity,

$$
\begin{aligned}
h(T) & =\sup _{\Gamma} h(\Gamma, T)=\sup _{\Gamma}\left\{h\left(\Gamma \cap M,\left.T\right|_{M}\right)+h\left(\Gamma \cap N,\left.T\right|_{N}\right)\right\} \\
& =\sup _{\boldsymbol{\Lambda}} h\left(\Lambda,\left.T\right|_{M}\right)+\sup _{\boldsymbol{\Theta}} h\left(\Theta,\left.T\right|_{N}\right)=h\left(\left.T\right|_{M}\right)+h\left(\left.T\right|_{N}\right) \text {. Q.E.D. }
\end{aligned}
$$

CoROLlARY. The analogous formula is true when the elementwise invariant partition is countable.

This follows from the more powerful theory developed in [3, Chapter 2] which deals with the difficulties that countable partitions raise. Every step follows exactly as above.

Lemma 1. There is an antiperiodic transformation on the unit square with any prescribed entropy.

Proof. Arbitrary Bernoulli shifts can be made conjugate to transformations on the unit square precisely in the same manner as 
the shift $B(1 / 2,1 / 2)$ is made conjugate to the Baker's transformation on the unit square. See [1] and [2] for more on Bernoulli shifts. Since conjugation preserves entropy [1, pp. 64-65], these transformations have entropy $-\sum_{i=1}^{i=n} p_{i} \log p_{i}$. Given $r>0$, there is an $n$, and $\left(p_{i}\right)_{i=1}^{i=n}$ such that $-\sum_{i=1}^{i=n} p_{i} \log p_{i}=r$. This follows from the intermediate value theorem and the fact that, for fixed $n$, the function

$$
f\left(p_{1}, \cdots, p_{n}\right)=-\sum_{i=1}^{i=n} p_{i} \log p_{i}
$$

subject to the constraints: $p_{i} \geqq 0, \sum p_{i}=1$, has a maximum equal to $\log n \rightarrow \infty$ as $n \rightarrow \infty$. For $r=\infty$, we conjugate the shift on countably many symbols,

$$
\begin{gathered}
B\left(1 / 2,1 / 4,1 / 16, \cdots, 1 / 2^{2^{n}}, \cdots, 1 / 2^{2^{n}}, \cdots\right), \\
2^{2^{n-n-1}} \text { times }
\end{gathered}
$$

to a transformation in the unit square.

All Bernoulli shifts are antiperiodic. Q.E.D.

The following lemma is used tacitly hereafter.

LeMma 2. If $E \subset I^{2}$ has positive measure, then $E$ is isomorphic to $I^{2}$ in the sense that there is a measurable, invertible measure-preserving bijection which preserves the normalized inherited measure.

This follows easily from Halmos' lemma [2, p. 74].

Notation. Let $T$ be a transformation. Define

$$
E_{n}=\left\{x: T^{n} x=x ; T^{j} x \neq x, 0 \leqq j<n\right\} ;
$$

$E_{\infty}=\left\{x: T^{n} x \neq x\right.$ for all $\left.n\right\}$. These form a countable measurable partition each element of which is $T$-invariant. If $\{A, B\}$ is a measurable partition, define $d_{A}(S, T)=m(\{x \in A: S x \neq T x\})$. Then $d(S, T)$ $=d_{A}(S, T)+d_{B}(S, T)$.

Proof of the Theorem. Let $T$ be a transformation. We distinguish two cases: either

(i) almost every point is periodic; or

(ii) the set of nonperiodic points has positive measure.

(i) By the corollary to the computation lemma, and the fact that periodic transformations have zero entropy, $h(T)=0$. Hence it suffices to find an invariant set of small measure: on this we may construct a transformation of arbitrary entropy by Lemma 1, and then apply the computation lemma. For some $n, m\left(E_{n}\right)>0$. Find a set $Y \subset E_{n}$ such that $\epsilon / n>m(Y)>0$. Then $Y \cup \cdots \cup T^{n-1} Y$ is invariant and of small enough measure. 
(ii) We reduce case (ii) to case (i) by use of the following fact: arbitrarily near any transformation $T$ is an $S$ which has almost every point periodic. The uniform approximation theorem of Halmos [2, p. 75] applied to $\left.T\right|_{E_{\infty}}: E_{\infty} \rightarrow E_{\infty}$, yields, for any $n$ and $\epsilon$, a $T^{\prime}: E_{\infty}$ $\rightarrow E_{\infty}$ which has period $n$ and is within $1 / n+\epsilon$ of $T$. Choosing $n$ large, defining $S$ to be $T$ on $I^{2}-E_{\infty}$ and to be $T^{\prime}$ on $E_{\infty}$, we have

$$
d(S, T)=d_{E_{\infty}}(S, T)+d_{I^{2}-E_{\infty}}(S, T)=1 / n+\epsilon+0<2 \epsilon \text {. Q.E.D. }
$$

Remark. From (ii) above it follows easily that periodic transformations are dense, a result used in [3, p. 103].

Proof. Take $S$ as above in (ii). If $S$ is periodic, we are done. If not, choose $N$ so that $\epsilon>m\left(\cup_{n>N} E_{n}\right)>0$. Then $S \mid \cup_{0 \leqq n \leqq N} E_{n}$ is periodic of period at most $N$ ! On $\bigcup_{n>N} E_{n}$, modify $S$ to be periodic with period $N$ ! The resulting transformation is periodic and within $3 \epsilon$ of an arbitrary $T$.

\section{REFERENCES}

1. Patrick Billingsley, Ergodic theory and information, Wiley, New York, 1965. MR 33 \#254.

2. P. R. Halmos, Lectures on ergodic theory, Chelsea, New York, 1956. MR 20 \#3958. 1969.

3. William Parry, Entropy and generators in ergodic theory, Benjamin, New York,

4. V. A. Rohlin, Lectures on entropy theory of transformations with invariant measure, Uspehi Mat. Nauk 22 (1967), no. 5 (137), 3-56 = Russian Math. Surveys 22 (1967), no. 5, 1-52. MR $36 \# 349$.

5. - Entropy of metric automorphism, Dokl. Akad. Nauk SSSR 124 (1959), 980-983. (Russian) MR 21 \#2037; errata, MR 21 p. 1599.

Columbia University, New York, New York 10027 